\title{
Multiplication is Discontinuous in the Hawaiian Earring Group (with the Quotient Topology) by
}

Paul FABEL

Presented by Czesaw BESSAGA

\begin{abstract}
Summary. The natural quotient map $q$ from the space of based loops in the Hawaiian earring onto the fundamental group provides a naturally occuring example of a quotient map such that $q \times q$ fails to be a quotient map. With the quotient topology, this example shows $\pi_{1}(X, p)$ can fail to be a topological group if $X$ is locally path connected.
\end{abstract}

1. Introduction. The Hawaiian earring $H E$ is the union of a null sequence of circles joined at a common point. If the fundamental group $\pi_{1}(H E, p)$ is endowed with a certain natural topology, we prove $\pi_{1}(H E, p)$ fails to be a topological group with the standard operations, and in the bargain obtain a naturally occurring example of a map $q: Y \rightarrow Z$ such that $q \times q: Y \times Y \rightarrow Z \times Z$ fails to be a quotient map.

Following the definitions in [2], there is a natural quotient topology one can impart on the familiar based fundamental group $\pi_{1}(X, p)$ of a topological space $X$.

If $L(X, p)$ denotes the space of $p$ based loops in $X$ with the compact open topology, and if $q: L(X, p) \rightarrow \pi_{1}(X, p)$ is the natural surjection, then we endow $\pi_{1}(X, p)$ with the quotient topology such that $A \subset \pi_{1}(X, p)$ is closed in $\pi_{1}(X, p)$ if and only of $q^{-1}(A)$ is closed in $L(X, p)$.

For spaces $X$ sufficiently simple on the small scale, $\pi_{1}(X, p)$ has the discrete topology and is certainly a topological group [7, 4], 3].

More generally Proposition 3.1 of [2] asserts that $\pi_{1}(X, p)$ is always a topological group with the familiar operations. However Tyler Lawson no-

2010 Mathematics Subject Classification: Primary 54G20; Secondary 54B15.

Key words and phrases: Hawaiian earring. 
ticed in 2006 that the proof of Proposition 3.1 depends on the questionable assumption that the product of the quotient maps $q \times q: L(X, p) \times L(X, p) \rightarrow$ $\pi_{1}(X, p) \times \pi_{1}(X, p)$ is again a quotient map (since for general topological spaces, if $q: Y \rightarrow Z$ is a quotient map then $q \times q: Y \times Y \rightarrow Z \times Z$ can fail to be a quotient map [13]).

Jeremy Brazas has recently found various examples of spaces $X$ such that $\pi_{1}(X, p)$ fails to have continuous multiplication [3]. Particular attention in [3] is given to spaces $X$ constructed in the following manner. Let $A$ be a totally disconnected subset of the positive real line, and consider $X$ as the union of planar circles of radius $1+a$, centered on the positive real line, and joined at the common point $(0,0)$. Such spaces $X$ are locally simply connected, but fail to be locally path connected. For example Brazas proves $\pi_{1}(X, p)$ fails to have continuous multiplication if $A$ is the positive rationals.

In contrast to the examples in [3], the Hawaiian earring $H E$ is locally path connected but not locally simply connected.

Various papers have referenced (or generalized) the false Proposition 3.1 of [2], including some of the author, and we will comment later in this paper on what can be discarded, safely ignored or possibly repaired.

As a consequence of Theorem 1 we know $\pi_{1}(H E, p)$ is a Hausdorff space but not a topological group with the familiar operations and this begs the question "Is $\pi_{1}(H E, p)$ regular?"

2. Main result and implications. The Hawaiian earring HE is the union of a null sequence of circles joined at a common point $p$.

Formally $H E$ is the following subspace of the plane $\mathbb{R}^{2}$. For an integer $n \geq 1$ let $X_{n}$ denote the circle of radius $1 / n$ centered at $(1 / n, 0)$ and define $H E=\bigcup_{n=1}^{\infty} X_{n}$.

Let $p=(0,0)$ and let $Y_{N}=\bigcup_{n=1}^{N} X_{n}$.

Let $R_{N}: H E \rightarrow Y_{N}$ denote the natural retraction collapsing $\bigcup_{n=N+1}^{\infty} X_{n}$ to $p$. The natural restriction between $Y_{N+1}$ and $Y_{N}$ determines an inverse limit space $\lim _{\leftarrow} Y_{N}$ such that $\lim _{\leftarrow} Y_{N}$ is canonically homeomorphic to $H E$ via $h: H E \rightarrow \lim _{\leftarrow} Y_{n}$ of the format $h(x)=(p, \ldots, p, x, x, \ldots)$. The map $h$ induces a continuous homomorphism $\phi: \pi_{1}(H E, p) \rightarrow \lim _{\leftarrow} \pi_{1}\left(Y_{N}, p\right)$, since in general maps between spaces induce continuous homomorphisms [2]. Of critical importance is the nontrivial fact that $\phi$ is one-to-one [12, [5], 6].

Let $L(H E, p)$ denote the space of maps $f:[0,1] \rightarrow H E$ such that $f(0)=$ $f(1)=p$, and endow $L(H E, p)$ with the compact open topology. Since $H E$ is a compact metric space, this is equivalent to the topology of uniform convergence.

Let $q: L(H E, p) \rightarrow \pi_{1}(H E, p)$ denote the canonical quotient map such that $q(f)=q(g)$ if and only if $f$ and $g$ are path homotopic in $H E$. 
Let $p_{n}=(2 / n, 0)$. Define the oscillation number $O_{n}: L(H E, p) \rightarrow$ $\{0,1,2,3,4, \ldots\}$ to be the maximum number $m$ such that there exists a set $T=\left\{0, t_{1}, \ldots, t_{2 m}\right\} \subset[0,1]$ such that $0<t_{1}<\cdots<t_{2 m}=1$ with $f\left(t_{2 i}\right)=p$ and $f\left(t_{2 i+1}\right)=p_{n}$.

REMARK 1. Fixing $n$ and $m$ and allowing $k$ to vary, suppose $f_{k} \rightarrow f$ uniformly in $L(H E, p)$ and $O_{n}\left(f_{k}\right) \geq m$ as shown by the sets $T_{k} \subset[0,1]$ such that $\left|T_{k}\right|=2 m+1$. Then if $T \subset[0,1]$ is a subsequential limit of $\left\{T_{k}\right\}$ in the Hausdorff metric, then $T$ shows $O_{n}(f) \geq m$.

Given $f \in L(H E, p)$, and natural numbers $m$ and $n$, we obtain a lower bound on $O_{n}(f)$ as follows. Suppose $f \in L(H E, p)$ and recall $R_{m}: H E \rightarrow Y_{m}$ is the natural retraction collapsing $X_{k}$ to $p$ for all $k>m$. If $n>m$ then $p_{n} \notin$ $Y_{m}$ (and hence $\left.O_{n}\left(R_{m}(f)\right)=0\right)$ and if $n \leq m$ then $O_{n}\left(R_{m}(f)\right)=O_{n}(f)$. Thus if $f_{1}=R_{m}(f)$ then $O_{n}\left(f_{1}\right) \leq O_{n}(f)$. Let $U$ be a contractible open subspace of $Y_{m}$ such that $p \in U$. Consider the open set $J=f_{1}^{-1}\left(Y_{m} \backslash\{p\}\right)$ with open interval components $J_{1}, J_{2}, \ldots$ Note $J \subset(0,1)$ and hence $\operatorname{diam}\left(J_{n}\right) \rightarrow 0$. Uniform continuity of $f$ ensures $\operatorname{diam}\left(f_{1}\left(J_{n}\right)\right) \rightarrow 0$. Thus, with finitely many exceptions, $f_{1}\left(\overline{J_{i}}\right) \subset U$. If $f_{1}\left(\overline{J_{i}}\right) \subset U$, replace $\left.f\right|_{\overline{J_{n}}}$ by the constant loop at $p$, to create a function $f_{2}:[0,1] \rightarrow Y_{m}$. If $J^{\wedge}$ denotes the union of intervals $J_{k}$ in $J$ such that $f_{1}\left(\overline{J_{i}}\right) \subset U$, the standard pasting lemma ensures $f_{2}=\left.\left.p\right|_{J^{\wedge}} \cup f_{1}\right|_{\left([0,1] \backslash J^{\wedge}\right)}$ is continuous, and applying a contraction of $U$ to $\left.f_{2}\right|_{\overline{J^{\wedge}}}$, we see $f_{2}$ is path homotopic to $f_{1}$ in $Y_{m}$. By construction $O_{n}\left(f_{2}\right)=O\left(f_{1}\right)$.

Recall $\pi_{1}\left(Y_{m}, p\right)$ is canonically isomorphic to $F_{m}$, the free group on $m$ generators $\left\{x_{1}, \ldots, x_{m}\right\}$ with letters $x_{i}$ corresponding to one counterclockwise orbit around the circle $X_{i}$. Let $w \in F_{m}$ denote the reduced finite word in $F_{m}$ corresponding to $\left[f_{2}\right] \in \pi_{1}\left(Y_{m}, p\right)$. Let $g:[0,1] \rightarrow Y_{m}$ be the unique path of constant Euclidean speed determined by the word $w$. Notice $O_{n}(g)$ is the total number of occurrences of $x_{n}$ and $x_{n}^{-1}$ in the word $w$. Thus if $v \in F_{m}$ denotes the (unreduced) finite word determined by $f_{2}$, then standard word reduction in $F_{m}$ from $v$ to $w$ (by successive deletion of consecutive inverse pairs $x_{i} x_{i}^{-1}$ or $\left.x_{j}^{-1} x_{j}\right)$ shows $|v| \geq|w|$. Note $R_{m}(f), f_{2}$ and $g$ are path homotopic in $Y_{m}$ and hence in $H E$. Thus in the particular case that $R_{m}(f)$ is path homotopic to $f$ in $H E$, the previous discussion is summarized in the following remark.

REMARK 2. Suppose $f$ and $g$ are in the same path component of $L(H E, p)$ and suppose $g:[0,1] \rightarrow Y_{m}$ is a path of constant speed corresponding to a maximally reduced finite word $w$ in the free group $F_{m}$ on $m$ letters. Then $O_{n}(f) \geq O_{n}(g)$.

REMARK 3. Since $\phi: \pi_{1}(H E, p) \rightarrow \lim _{\leftarrow} \pi_{1}\left(Y_{n}, p\right)$ is continuous and one-to-one, and since $\lim _{\leftarrow} \pi_{1}\left(Y_{n}, p\right)$ is a $T_{2}$ space, the space $\pi_{1}(H E, p)$ is $T_{2}$. In particular $\pi_{1}(H E, p)$ is $T_{1}$ and hence the path components of $L(H E, p)$ are closed subspaces of $L(H E, p)$. 
REMARK 4. If $Z$ is a metric space such that each path component of $Z$ is a closed subspace of $Z$, then each path component of $Z \times Z$ is a closed subspace of $Z \times Z$. (If $\left(x_{n}, y_{n}\right) \rightarrow(x, y)$ and $\left\{\left(x_{n}, y_{n}\right)\right\}$ is in a path component of $Z \times Z$ then obtain paths $\alpha$ and $\beta$ in $Z$ connecting $x$ to $\left\{x_{n}\right\}$ and $y$ to $\left\{y_{n}\right\}$ and $(\alpha, \beta)$ is the desired path in $Z \times Z$.)

TheOrem 1. The product of quotient maps $q \times q: L(H E, p) \times L(H E, p)$ $\rightarrow \pi_{1}(H E, p) \times \pi_{1}(H E, p)$ fails to be a quotient map, standard multiplication (by path class concatenation) $M: \pi_{1}(H E, p) \times \pi_{1}(H E, p) \rightarrow \pi_{1}(H E, p)$ is discontinuous, and the fundamental group $\pi_{1}(H E, p)$ fails to be a topological group with the standard group operations.

Proof. Let $x_{n} \in L(H E, p)$ orbit $X_{n}$ once counterclockwise.

Applying path concatenation, for integers $n \geq 2$ and $k \geq 2$ and $n \neq k$ let $a(n, k) \in L(H E, p)$ be a based loop corresponding to the finite word $\left(x_{n} x_{k} x_{n}^{-1} x_{k}^{-1}\right)^{k+n}$ and let $w(n, k) \in L(H E, p)$ be a based loop corresponding to the finite word $\left(x_{1} x_{k} x_{1}^{-1} x_{k}^{-1}\right)^{n}$.

Let $F \subset \pi_{1}(H E, p) \times \pi_{1}(H E, p)$ denote the set of all doubly indexed ordered pairs $([a(n, k)],[w(n, k)])$.

Let $P \in L(H E, p)$ denote the constant map such that $f([0,1])=\{p\}$.

To prove $q \times q$ fails to be a quotient map it suffices to prove that $F$ is not closed in $\pi_{1}(H E, p) \times \pi_{1}(H E, p)$ and $(q \times q)^{-1}(F)$ is closed in $L(H E, p) \times$ $L(H E, p)$.

To prove $F$ is not closed in $\pi_{1}(H E, p) \times \pi_{1}(H E, p)$ we will prove that $([P],[P]) \notin F$ but $([P],[P])$ is a limit point of $F$.

Recall $\phi: \pi_{1}(H E, p) \rightarrow \lim _{\leftarrow} \pi_{1}\left(Y_{m}, p\right)$ is one-to-one and $k \geq 2$. Thus $[P] \neq[w(n, k)]$ and $[P] \neq[a(n, k)]$. Thus $([P],[P]) \notin F$.

Suppose $[P] \in U$ and $U$ is open in $\pi_{1}(H E, p)$. Let $V=q^{-1}(U)$. Then $V$ is open in $L(H E, p)$ since, by definition, $q$ is continuous.

Note $P \in V$. Thus there exist $N$ and $K$ such that if $n \geq N$ and $k \geq K$ then $a(n, k) \in V$. Note $\left(x_{1} x_{1}^{-1}\right)^{N}$ is path homotopic to $P$ and hence $\left(x_{1} x_{1}^{-1}\right)^{N} \in V$. Observe that $w(N, k)$ (suitably parameterized over $[0,1]$ ) converges to $\left(x_{1} x_{1}^{-1}\right)^{N}$ uniformly in $L(H E, p)$. Thus there exists $K_{2} \geq K$ such that if $k \geq K_{2}$ then $w(N, k) \in V$. Hence $\left(\left[w\left(N, K_{2}\right)\right],\left[a\left(N, K_{2}\right)\right]\right) \in U \times U$. This proves $([P],[P])$ is a limit point of $F$, and thus $F$ is not closed in $\pi_{1}(H E, p) \times \pi_{1}(H E, p)$.

To prove $(q \times q)^{-1}(F)$ is closed in $L(H E, p) \times L(H E, p)$ suppose that $\left(f_{m}, g_{m}\right) \rightarrow(f, g)$ uniformly and $\left(f_{m}, g_{m}\right) \in(q \times q)^{-1}(F)$. Note $O_{1}(w(n, k))$ $=2 n$ and $O_{N}(a(N, k)) \geq 2(N+k)$.

Let $a\left(n_{m}, k_{m}\right)$ and $w\left(n_{m}, k_{m}\right)$ be path homotopic to respectively $f_{m}$ and $g_{m}$.

By Remark 2, $O_{1}\left(g_{m}\right) \geq O_{1}\left(w\left(n_{m}, k_{m}\right)\right)=2 n_{m}$. 
Thus if $\left\{n_{m}\right\}$ contains an unbounded subsequence then, by Remark 1 . $O_{1}(g) \geq \limsup O_{1}\left(w\left(n_{m}, k_{n_{m}}\right)\right)=\infty$ and we have a contradiction since $O_{1}(g)<\infty$. Thus $\left\{n_{m}\right\}$ is bounded and so takes on finitely many values.

In similar fashion, if $\left\{k_{m}\right\}$ is unbounded then there exists $N$ and a subsequence $\left\{k_{m_{l}}\right\}$ such that $O_{N}\left(a\left(N, k_{m_{l}}\right)\right) \rightarrow \infty$. It follows that $O_{N}(f) \geq$ $\lim \sup O_{N}\left(a\left(N, k_{m_{l}}\right)\right)=\infty$, contradicting the fact that $O_{N}(f)<\infty$.

Thus both $\left\{n_{m}\right\}$ and $\left\{k_{m}\right\}$ are bounded and hence (by the pigeon hole principle) there exists a path component $B \subset L(H E, p) \times L(H E, p)$ containing a subsequence $\left(f_{m_{l}}, g_{m_{l}}\right)$.

It follows from Remarks 3 and 4 that $(f, g) \in B$. Thus $(q \times q)^{-1}(F)$ is closed and hence $q \times q$ fails to be a quotient map.

In similar fashion we will prove that group multiplication $M: \pi_{1}(H E, p)$ $\times \pi_{1}(H E, p) \rightarrow \pi_{1}(H E, p)$ is discontinuous, and hence $\pi_{1}(H E, p)$ will fail to be a topological group with the standard group operations. To achieve this we will exhibit a closed set $A \subset \pi_{1}(H E, p)$ such that $M^{-1}(A)$ is not closed in $\pi_{1}(H E, p) \times \pi_{1}(H E, p)$.

Consider the doubly indexed set $A=M(F) \subset \pi_{1}(H E, p)$ such that each element of $A$ is of the form $[a(n, k)] *[w(n, k)]$ (with $*$ the denoting familiar path class concatenation).

On the one hand observe by definition (and since $\phi$ is one-to-one) $[a(n, k)]$ $*[w(n, k)] \neq[P]$. Thus $[P] \notin A$ and $([P],[P]) \notin M^{-1}(A)$. Note $F \subset M^{-1}(A)$ and by the previous argument $([P],[P])$ is a limit point of $F$. Thus $M^{-1}(A)$ is not closed in $\pi_{1}(H E, p) \times \pi_{1}(H E, p)$.

On the other hand we will prove $A$ is closed in $\pi_{1}(H E, p)$ by proving $q^{-1}(A)$ is closed in $L(H E, p)$. Suppose that $f_{m} \rightarrow f \in L(H E, p)$ and $f_{m} \in q^{-1}(A)$.

Obtain $n_{m}$ and $k_{m}$ such that $f_{m} \in\left[a\left(n_{m}, k_{m}\right)\right] *\left[w\left(n_{m}, k_{m}\right)\right]$.

In similar fashion to the previous proof, if $\left\{n_{m}\right\}$ is unbounded we obtain the contradiction $O_{1}(f) \geq \limsup O_{1}\left(f_{m}\right)=\infty$.

If $\left\{n_{m}\right\}$ is bounded and $\left\{k_{m}\right\}$ is unbounded we obtain $N$ and a subsequence $k_{m_{l}}$ and the contradiction $O_{N}(f) \geq \lim \sup O_{N}\left(f_{m_{l}}\right)=\infty$.

Thus both $\left\{n_{m}\right\}$ and $\left\{k_{m}\right\}$ are bounded. It follows by the pigeon hole principle that some path component $B \subset L(H E, p)$ contains a subsequence $\left\{f_{m_{l}}\right\}$ and Remark 3 implies that $f \in B$. Hence $q^{-1}(A)$ is closed in $L(H E, p)$ and thus $A$ is closed in $\pi_{1}(H E, p)$.

Theorem 1 contradicts some published claims to the contrary and we offer brief assessment of how this affects various published results.

It is falsely claimed in 2], [1 that group multiplication in $\pi_{1}(X, p)$ is continuous and that $\pi_{1}(X, p)$ is a topological group. However these mistakes do not appear to directly affect arguments elsewhere in the papers (some of which have also been challenged [10]). 
The introduction in [10] mentions that $\pi_{1}(X, p)$ is a topological group, but apparently none of the results or proofs are affected by this remark.

Theorem 2 of [9] claims that $\pi_{1}(X, p)$ is regular iff $\pi_{1}(X, p)$ is a $T_{1}$ space. This claim is suspicious since the proof assumes (incorrectly) that $\pi_{1}(X, p)$ is a topological group, and Example 4.22 of [3] shows the $T_{1}$ property of $\pi_{1}(X, p)$ does not guarantee that $\pi_{1}(X, p)$ is completely regular.

The paper [8] develops a false generalization (Theorem 2.7) of Proposition 3.1 of [2] and the proof makes the common mistake of falsely assuming that the product of quotient maps is a quotient map. However the main application (the validity of Theorem 3.12 which constructs a completion $\overline{B_{\infty}}$ of the infinite braid group) in [8] is unlikely to be affected (since with the quotient topology, the pure braid subgroup of $\overline{B_{\infty}}$ is the topological inverse limit of the finite pure braid groups, and hence a topological group). Nevertheless it would be appropriate to provide a new and careful argument that multiplication in $\overline{B_{\infty}}$ is continuous.

In [14, Lemma 1.1 and its proof assert (falsely) that $\pi_{1}(X, p)$ is a topological group. In [11], Theorem 2.1 and its proof assert (falsely) that $\pi_{n}(X, p)$ is a topological group for all $n$, and the familiar mistake is to assume that the product of quotient maps is a quotient map.

3. Summary. The Hawaiian earring $H E$ is a locally path connected compact metric space whose fundamental group $\pi_{1}(H E, p)$ is shown in this paper to have discontinuous group multiplication with a certain natural topology on $\pi_{1}(H E, p)$. The topology of $\pi_{1}(H E, p)$ is the quotient topology inherited under the natural map from the space of $p$-based loops in $H E$.

\section{References}

[1] D. K. Biss, A generalized approach to the fundamental group, Amer. Math. Monthly 107 (2000), 711-720.

[2] - , The topological fundamental group and generalized covering spaces, Topology Appl. 124 (2002), 355-371.

[3] J. Brazas, The topological fundamental group and free topological groups, ibid. 148 (2011), 779-802.

[4] J. S. Calcut and J. D. McCarthy, Discreteness and homogeneity of the topological fundamental group, Topology Proc. 34 (2009), 339-349.

[5] J. W. Cannon and G. R. Conner, The combinatorial structure of the Hawaiian earring group, Topology Appl. 106 (2000), 225-271.

[6] B. de Smit, The fundamental group of the Hawaiian earring is not free, Int. J. Algebra Comput. 2 (1992), 33-37.

[7] P. Fabel, Metric spaces with discrete topological fundamental group, Topology Appl. 154 (2007), 635-638.

[8] -, Completing Artin's braid group on infinitely many strands, J. Knot Theory Ramif. 14 (2005), 979-991. 
[9] P. Fabel, A retraction theorem for topological fundamental groups with application to the Hawaiian earring, Topology Appl. 154 (2007), 722-724.

[10] - , The topological Hawaiian earring group does not embed in the inverse limit of free groups, Algebr. Geom. Topol. 5 (2005), 1585-1587.

[11] H. Ghane, Z. Hamed, B. Mashayekhy, and H. Mirebrahimi, Topological homotopy groups, Bull. Belg. Math. Soc. Simon Stevin 15 (2008), 455-464.

[12] J. W. Morgan and I. Morrison, A van Kampen theorem for weak joins, Proc. London Math. Soc. (3) 53 (1986), 562-576.

[13] J. Munkres, Topology, 2nd ed., Prentice-Hall, 1999.

[14] H. Sahleh and A. G. Sanatee, Embedding of the fundamental topological group in the inverse limit of discrete groups, Int. J. Contemp. Math. Sci. 3 (2008), 305-310.

Paul Fabel

Department of Mathematics and Statistics

Mississippi State University

Drawer MA, Mississippi State, MS 39762, U.S.A.

E-mail: fabel@ra.msstate.edu

http://www2.msstate.edu/ fabel/

Received November 17, 2009;

received in final form April 3, 2011 
